\title{
MicroMeeting
}

\section{Cell shape, division and development: the 2002 American Society for Microbiology (ASM) conference on prokaryotic development}

\author{
Rainer M. Figge ${ }^{1 *}$ and James W. Gober ${ }^{2}$ \\ ${ }^{1}$ Aventis Pharma, Functional Genomics, Bât. Magendie, \\ labo 123, 13, quai Jules Guesde, 94400 Vitry-sur-Seine, \\ France. \\ ${ }^{2}$ Department of Chemistry and Biochemistry and the \\ Molecular Biology Institute, University of California, Los \\ Angeles, CA 90095-1569, USA.
}

\section{Summary}

In the last decade, the use of cytological techniques, together with the analysis of complete genomes, has dramatically advanced our understanding of bacterial development. Work on several well-developed model systems such as Bacillus subtilis, Caulobacter crescentus, Myxococcus xanthus and Streptomyces spp., has provided us with an in-depth understanding of processes such as sporulation, multicellular behaviour and the bacterial cell cycle. At the same time, these studies have revolutionized our view of the bacterial cell and shown it to be a highly complex entity with spatial and temporal organization. The recent American Society for Microbiology (ASM) conference on prokaryotic development demonstrated that several laboratories have now started to connect data obtained through functional genomic analysis with subcellular organization, thereby generating threedimensional regulatory networks. This meeting report highlights new findings in the field, such as regulation of protein localization during sporulation and the cell cycle, control of cell-cell interaction and the initiation of cell division.

\section{Introduction}

Initially, the terms bacteria and development do not seem to go together. Rather, development is something that we

Accepted 26 November, 2002. *For correspondence. E-mail Rainer.Figge @ aventis.com; Tel. (+33) 1589383 19; Fax (+33) 158 932625.

(C) 2003 Blackwell Publishing Ltd associate with multicellular organization. Nevertheless, some bacterial species are capable of undergoing highly elaborate developmental processes, often differentiating from one cell type to another in a systematic manner. Why have these seemingly simple organisms adopted such complex life styles? The answer lies, in part, in the ever and rapidly changing environmental conditions confronted by these bacteria. Development allows certain bacteria to survive extreme environmental conditions such as starvation, heat and strong acids. Differentiation during the cell cycle guarantees maximal distribution in the environment. Additionally, genetic exchange or competence as part of a developmental programme ensures that important traits are dispersed throughout the population.

Over the last 30 years, several model organisms have been used to study bacterial development. The most prominent among these are Bacillus subtilis, Caulobacter crescentus, Myxococcus xanthus and Streptomyces coelicolor. The release of the complete bacterial genome sequences (Kunst et al., 1997; Nierman et al., 2001; Bentley et al., 2002; Myxococcus, Monsanto, http:// microbial.cereon.com/) of these organisms enables us to study developmental processes by global expression analyses at the gene and protein levels. Together with traditional genetics, these tools are revealing molecular regulatory networks underlying developmental processes. Similarly, the introduction of cytological techniques into bacterial research has rapidly advanced our understanding of these fascinating organisms. In particular, studies on bacterial development have revealed that bacteria are highly organized: chromosomes and certain proteins move in a dynamic and precise way within the cell. Several fundamental mechanisms underlying development, such as compartmentalized gene expression and protein localization, have emerged and been proved to be similar to those operating in eukaryotic organisms.

The previous meeting that concentrated on the development of unicellular organisms was the Harden Conference held in Cirencester, UK, in 1996. The programme of this meeting and its predecessor, a Keystone Conference in Durango, USA, in 1993, presented a balanced mixture 
of both prokaryotic and eukaryotic developmental systems. In the few years after 1996, there has been an explosion in the number of laboratories and of the techniques used and, with them, new concepts and paradigms in bacterial growth and development. Thus, bacterial development, as a field, has come into its own. With this in mind, Y. Brun (Indiana University) and L. Shimkets (University of Georgia) organized this year's ASM meeting on prokaryotic development, which took place in Quebec City, Canada, on 10-14 July. This report gives an overview of the most recent discoveries in the field of bacterial development. It includes new data on cell shape, on the localization of regulatory proteins and on cell-cell signalling. A series of MicroReviews to be published shortly will give more specific details on three well-studied developmental systems in Myxococcus, Streptomyces and Escherichia coli.

\section{Bacillus, master of sporulation}

Under conditions of nutrient depletion at high cell density, $B$. subtilis undergoes a developmental programme leading to the formation of heat- and desiccation-resistant endospores. Several input signals are integrated in a phosphorelay of sensor kinases and response regulators, culminating in the phosphorylation of SpoOA, the master regulator transcription factor that directs the initiation of sporulation (reviewed by Burkholder and Grossman, 2000). Kinase activity is counteracted by protein phosphatases of the Rap and SpoOE families that respond to conditions favouring vegetative growth over sporulation. M. Perego (Scripps Research Institute, La Jolla, CA, USA) reported on the mechanisms that regulate the activity of RapA and RapC by specific pentapeptide inhibitors (Perego and Brannigan, 2001). RapA phosphatase activity on SpoOF $\sim P$ is inhibited in vivo by a pentapeptide generated from the phrA gene. In vitro, addition of this pentapeptide dissolves the otherwise stable complex of SpoOF P and RapA (Ishikawa et al., 2002). Interestingly, the RapA homologue, RapC, does not regulate its target response regulator, ComA, by dephosphorylation. Instead, RapC binds to unphosphorylated ComA and prevents it from binding to its target promoters, which, in turn, can be reversed by the cognate pentapeptide.

Spo0A directly activates the expression of early sporulation genes and is required for polar septum formation mediated by a switch in the localization of FtsZ (Levin and Losick, 1996). S. Ben-Yehuda (R. Losick's laboratory, Harvard University, Cambridge, MA, USA) and R. Losick showed that medial FtsZ rings are replaced by spiralshaped filaments of FtsZ that grow bidirectionally away from the cell centre to redeploy FtsZ to the poles. The formation of spirals and, subsequently, polar FtsZ rings depends largely on an increase in FtsZ transcription and the activation of the gene encoding the FtsZ-associated membrane protein SpollE (Ben-Yehuda and Losick, 2002).

G. Real (A. O. Henriques' laboratory, Instituto de Tecnologia Quimica e Biologica, Oeiras, Portugal) has found a link between the chromosome segregation proteins Spo0J/Soj and the cell division protein DivlB. A divIB mutation was identified that reduces the levels of DivIB, prevents transcription of spol/G and polar septum formation, and mislocalizes Spo0J-GFP. Deletion of the spooJsoj locus in the DivIB mutant restores polar septum formation and, thus, sporulation, even though the abundance of DivIB remains low. This indicates that high levels of DivIB are required for a regulatory role on the Spo0J/Soj system.

During sporulation, the formation of the polar septum creates two distinct, cellular compartments that are characterized by differential gene expression and specific subcellular protein localization. A. A. Rubio (K. Pogliano's laboratory, University of California San Diego, La Jolla, CA, USA) finds that, in $B$. subtilis, initial targeting of heterologous green fluorescent protein (GFP) fusions to the polar septum only depends on forespore-specific expression through a $\sigma^{F}$ promoter and membrane-spanning segments. In contrast to the heterologous proteins, GFPSpolIQ, a GFP fusion to a native forespore-specific membrane protein, moves around the forespore in distinct steps. This movement depends on proteins used for septal thinning (SpolIM, SpolIP and SpolID), which are made in the mother cell. Whereas MalF $_{12}$-GFP (membranespanning segments 1 and 2 from $E$. coli MalF) does not remain in the septal membrane, GFP-SpollQ was retained there by an unidentified mother cell protein. Thus, it is likely that, initially, all membrane proteins are inserted into the septal membrane. Subsequently those specifically involved in sporulation are trapped by other proteins or peptidoglycan and cannot diffuse as freely as the heterologous membrane proteins. Protein localization of mother cell-specific proteins during a later stage in sporulation was investigated by C. van Ooji (R. Losick's laboratory). SpoVM and YabP localized in different ways during sporulation. The localization pattern of both proteins was $\sigma^{\mathrm{E}}$, but not $\sigma^{\mathrm{F}}$, dependent, indicating that a mother cell protein may be required for their localization. SpollIE, a protein required for chromosome translocation into the forespore during sporulation in $B$. subtilis, is, through an unknown mechanism, also essential for proper compartmentalization of $\sigma^{\mathrm{F}}$ activity to the forespore and $\sigma^{\mathrm{E}}$ activity to the mother cell. R. Dutton (K. Pogliano's laboratory, University of California San Diego) finds that SpollIE acted as a physical barrier to the diffusion of soluble proteins between the mother cell and the forespore. This suggests that SpollIE may play a septin-like role between the two cells during chromosome translocation, thereby 
preventing cytoplasmic proteins such as $\sigma^{F}$ and $\sigma^{E}$ from diffusing into the wrong cellular compartment.

Expression of specific late sporulation genes in the mother cell depends on the activation of $\sigma^{\mathrm{K}}$. Expression of $\sigma^{\mathrm{K}}$ in $B$. subtilis is preceded by a recombination event that fuses the $\mathrm{N}$ - and $\mathrm{C}$-terminal portions of $\sigma^{\mathrm{K}}$ (Kunkel et al., 1990). J. D. Haraldsen (A. L. Sonenshein's laboratory, Tufts University, School of Medicine, Boston, MA, USA) has characterized the $\sigma^{\mathrm{K}}$ gene in different Clostridium difficile strains. Similar to B. subtilis, $C$. difficile $\sigma^{k}$ is interrupted by a large insertion that is excised specifically in the mother cell during sporulation. As $C$. difficile strains lacking the insertion show a sporulation defect and $\sigma^{\mathrm{K}}$ of $C$. difficile lacks the processing region that controls $\sigma^{\mathrm{K}}$ activity in $B$. subtilis, it is likely that excision has a regulatory function in $C$. difficile.

D. B. Kearns (R. Losick's laboratory) investigated swarming motility in a wild $B$. subtilis strain. Swarming required cell-cell contact and was density dependent. Swarming monolayers differentiated into two populations, an outer circle of cells with many flagella and compact nucleoids, and an inner circle of smaller cells with less compact nucleoids and few flagella. Swarming depends on the presence of the flagellin gene hag and the sifA gene encoding a lipopeptide surfactant.

\section{Biofilms}

Highly structured, surface-associated communities of bacteria, known as biofilms, play an important role in industrial processes and in the colonization of hosts by pathogens. Recently Branda et al. (2001) showed that $B$. subtilis can form complex biofilms that produce fruiting bodies used for sporulation. S. Branda (R. Kolter's laboratory, Harvard Medical School, Boston, MA, USA) demonstrated how transcriptional regulators controlling entry into sporulation and production of biosurfactant are required for biofilm formation. Mutations in a gene probably involved in the production of the extracellular matrix $(y h \times B)$ and in genes that may be required for cell-cell signalling (ecsD, sipW and yqeK) cause defects in biofilm formation. N. R. Stanley (B. Lazazzera's laboratory, UCLA, Los Angeles, CA, USA) performed a microarray analysis on $B$. subtilis biofilms and showed that $6 \%$ of all genes are differentially regulated during biofilm formation. Analysis of these genes revealed that glucose negatively regulates biofilm formation through the catabolite control protein, CcpA. Flagellar and glycolytic genes were downregulated, and bacteriophage and ribosome encoding genes were upregulated in biofilms. These findings correlate with the expression profiles found in Pseudomonas aeruginosa biofilms (Whiteley et al., 2001). T. Mah (G. O'Toole's laboratory, Dartmouth Medical school, Hanover, $\mathrm{NH}$, USA) has isolated $P$. aeruginosa mutants that still form biofilms but are highly sensitive to the antibiotic aminoglycoside tobramycin. Analysis of the mutants may lead to an understanding of the high resistance level against antibiotics usually observed in biofilms. N. Bomchil (R. Kolter's laboratory, Harvard Medical school, Boston, MA, USA) has identified a new gene, $m b a A$, that is important for the maintenance of biofilm architecture by Vibrio cholerae. It harbours EAL and a GGDEF domain, which are present in some sensor/regulator proteins (e.g. controlling polysaccharide biosynthesis).

M. Chaplan (S. Hultgren's laboratory, Washington University, St Louis, MO, USA) investigated a uropathogenic E. coli strain that forms egg-shaped communities in mice bladders. Expression and secretion of curli fibre proteins that form fibres that resemble Alzheimer's amyloid are a prerequisite for the generation of this biofilm and, thus, for infection. A new secretion system consisting of two chaperones (CsgE, CsgF) and a pore protein (CsgG) transports the fibre monomer (CsgA) outside the cell, where it assembles.

\section{Regulation of the cell cycle in Caulobacter crescentus}

Caulobacter crescentus undergoes a developmental programme as part of its cell cycle. A motile swarmer cell sheds its flagellum, grows a stalk used for attachment and starts producing swarmer cells (England and Gober, 2001). Caulobacter is an excellent model system for studying cell cycle events, as synchronized populations are easily obtained. L. Shapiro (Stanford University, Stanford, CA, USA) showed that the execution of specific cell cycle events correlates with the hierarchical expression of the required genes. The pilus-specific secretory apparatus $\mathrm{CpaE} / \mathrm{F}$ is assembled first, and the pilin, PilA, is made last. CtrA, the master regulator of the Caulobacter cell cycle, was recently shown to regulate 168 genes. Using a chromosome immunoprecipitation assay, Shapiro and coworkers have now identified 53 loci that are directly regulated by CtrA (Laub et al., 2002). A phosphorelay system that culminates in the phosphorylation of CtrA processes cell cycle cues, allowing co-ordinated progression of the cell cycle. C. Jacobs (Yale University, New Haven, CT, USA) studied the spatial localization of the phosphorelay kinases PleC and DivJ and the response regulator DivK (Jensen et al., 2002). The localization of DivK depends on its phosphorylation by DivJ and probably dephosphorylation by the sensor kinase PleC. In Sinorhizobium meliloti, the DivK homologue also acts as a cell cycle regulator and is polarly localized, indicating that cell cycle progression in S. meliloti may be similar to Caulobacter. A. Newton (Princeton University, Princeton, NJ, USA) and coworkers have performed a yeast two-hybrid screen to identify novel interacting partners of DivK. Apart from the kinases DivJ, PleC and DivL, two new kinases, CckN and 
CckO, were shown to interact with DivK. P. H. Viollier (from L. Shapiro's laboratory, Stanford University, Stanford, CA, USA) and A. J. Hinz (from Y. V. Brun's laboratory, Indiana University, Bloomington, IN, USA) have both shown that PodJ, a pleiotropic regulatory protein, localizes to the swarmer pole and plays an important role in pilus formation. In addition, PodJ is required for the localization of PleC (Hinz et al., 2002; Viollier et al., 2002).

J. W. Gober (UCLA, Los Angeles, CA, USA) showed that FliX, previously identified as a structural gene in flagellar biogenesis (Mohr et al., 1998), is a regulatory protein that acts on the $\sigma^{54}$-dependent regulator, $\mathrm{FlbD}$, in a positive and negative way, depending on the assembly status of the flagellum (Muir et al., 2001). Interestingly, FliX regulates FlbD through a direct interaction that does not involve phosphorylation, a phenomenon that has not been reported for a $\sigma^{54}$ activator protein containing a receiver domain. Both proteins also regulate the completion of cell division, as observed by R. Muir (J. W. Gober's laboratory, UCLA, Los Angeles, CA, USA) (Muir and Gober, 2001). Thus, the progression of flagellar assembly and the completion of cell division are coupled. E. M. Quardokus (Y. V. Brun's laboratory, Indiana University, Bloomington, IN, USA) found that a mutant of the $\sigma^{54}$ dependent response regulator TacA is stalkless and exhibits aberrant cell division. A stalk is generated at each pole under phosphate starvation. In the tacA mutant, some critical regulators of development exhibit an altered localization or are not localized. These data, together with studies on the inhibition of cell division, indicate that TacA is required for the operation of a checkpoint that represses polar development when cell division is blocked.

L. E. West (L. Shapiro's laboratory, Stanford University, Stanford, CA, USA) has extended the cell cycle transcriptome analysis of Caulobacter to the entire genome. Several groups of genes were shown to be transcriptionally regulated upon entry into stationary phase or when the cells were starved for carbon during mid-log phase. The enormous amounts of data generated by functional genomics require the development of new bioinformatics tools. S. C. Chen (H. H. McAdams' laboratory, Stanford University, Stanford, CA) presented a new package for genome sequence analysis, called GENOME TOOLS. GENOME TOOLS consists of several programs and a user interface facilitating access to genome sequence information. It is accessible at http://genome-tools.sourceforge.net. K. Keiler (Pennsylvania State University, University Park, PA, USA) showed that the small tmRNA, SsrA, is required for the control of the Caulobacter cell cycle. Temporal transcription and degradation controls SsrA abundance. SsrA RNA possesses properties of both transfer and messenger RNA and interacts with ribosomes to target nascent polypeptides for degradation, an activity required for the $\mathrm{G} 1-\mathrm{S}$ transition.
M. Ackermann (U. Jenal's laboratory, Biozentrum, University of Basel, Basel, Switzerland) has used Caulobacter to study the connection between evolution and development. Three independent liquid cultures were grown for 7000 generations. In all populations, growth rates increased rapidly during the first 1000 generations through a reduction in the swarmer phase of the cell cycle. The populations acquired different morphological changes unique to each culture. Prolonged growth resulted in the appearance of mutations (e.g. pleC) that, when introduced into wild-type cells, decreased the fitness of the strain. Analysis of some of the mutants that have lost asymmetry might answer the question of how asymmetry is generated in Caulobacter.

\section{Chromosome/plasmid partitioning, cell division and cell shape}

Chromosome and plasmid partitioning are an important part of the cell cycle, ensuring that genetic information is properly distributed to the two daughter cells. Recently, the partitioning proteins ParA and ParB and the partitioning site parS, originally described in the phage $\mathrm{P} 1$ and $\mathrm{F}$ plasmids, have been shown to play an important role in chromosome segregation (Draper and Gober, 2001). The analogous system used in the partitioning of the Escherichia coli plasmid, R1, requires the DNA-binding protein ParR, the ATPase ParM and the cognate binding sequence parC for proper segregation. J. Møller-Jensen (K. Gerdes' laboratory, SDU-Odense University, Odense, Denmark) has investigated the role of ParM in this process. ParM forms dynamic, actin-like filaments. Polymerization of ParM requires ParR/parC as a nucleation point and generates the force needed to move plasmids to opposite cell poles (Møller-Jensen et al., 2002). The structure of ParM, which was solved together with the group of J. Lowe (MRC-LMB, Cambridge, UK), closely resembles the structures of actin and the bacterial actin homologue MreB. Chromosome partitioning and cell division are tightly coupled in Caulobacter (Mohl et al., 2001). J. Easter (J. W. Gober's laboratory, UCLA, Los Angeles, CA, USA) showed that the chromosome partitioning proteins ParA and ParB couple chromosome partitioning with cell division through a novel molecular switch. ParB, which is reminiscent of the exchange factors for eukaryotic G-proteins, regulates the ATPase activity of ParA by promoting nucleotide exchange. In vitro, the ParB DNA-binding protein can be displaced from its binding site by the addition of ParA-ATP, but not of ParA-ADP. ParA-ADP possesses single-strand DNA-binding activity and might repress the transcription of genes implicated in early cell division. It is likely that the decrease in the fraction of ParA-ADP versus ParA-ATP, as a consequence of partitioning of the CoriParB complexes to opposite cell poles, releases a ParA- 
ADP imposed cell division block (Easter and Gober, 2002).

In contrast to Caulobacter, in which cell division is regulated, at least in part, by the ParA and ParB system, placement of the division site in $E$. coli is regulated by nucleoid occlusion and the min system (Margolin, 2001). The Min system consists of three proteins: MinC, the actual inhibitor of FtsZ assembly; MinD, a member of the ParA ATPase family; and MinE, a topology factor for MinC and MinD activity. MinC and MinD oscillate from pole to pole in a MinE-dependent manner. Thus, MinE restricts MinCD to the polar regions of the cell, allowing FtsZ polymerization at the centre. P. de Boer (Case Western Reserve University School of Medicine, Cleveland, $\mathrm{OH}$, USA) and J. Lutkenhaus (University of Kansas Medical Center, Kansas City, KS, USA) have studied the interactions between Min proteins in the presence of phospholipids (PL) in vitro. Both showed that MinD binds to PL and that binding is enhanced in the presence of ATP (Hu et al., 2002). MinE binding to MinD is dependent on the presence of PL. J. Lutkenhaus observed that the carboxylterminal tail of MinD, which is highly conserved in several bacterial species, is required for membrane binding. MinE stimulates the ATPase activity of MinD, which is released from the membrane upon ATP cleavage. In contrast, release of MinC from the complex is independent of the MinD ATPase activity and precedes the release of MinD. P. de Boer showed that, in the presence of the slowly hydrolysed ATP analogue, ATP $\gamma \mathrm{S}$, MinC that was bound to MinD-coated vesicles could still be released, in contrast to MinD that remained bound.

A prerequisite for the functioning of the Min system is the rod shape of $E$. coli. Penicillin binding protein 2 (PBP2), RodA and the Mre proteins all play an important role in maintaining the rod shape. Recently, Jones et al. (2001) showed that, in B. subtilis, the two actin-like proteins MreB and $\mathrm{Mbl}$ form spirals that regulate cell width and determine the linear axis of cell growth respectively. The crystal structure of Thermotoga maritima MreB revealed an actin fold, proving MreB to be a true actin homologue (van den Ent et al., 2001). J. Errington (Sir William Dunn School of Pathology, University of Oxford, Oxford, UK) showed that Mbl forms paired helical filaments similar to the actin cables of yeast cells. These cables turn over within 5-10 min and are continuously remodelled, as demonstrated by fluorescence recovery after photobleaching. Filaments grow equally in both directions and are interrupted at cell division. Using fluorescently labelled vancomycin, which binds to terminal DAla-D-Ala chains of nascent peptidoglycan, Errington and coworkers found spiral-like structures similar to the $\mathrm{Mbl}$ spirals. Depletion of MurE, the diaminopimelic acid-adding enzymes in peptidoglycan biosynthesis, and the addition of bacitracin, which inhibits the transport of murein pre- cursors, abolishes the labelling of the spiral structures by vancomycin. R. M. Figge (J. W. Gober's laboratory, UCLA, Los Angeles, CA, USA) has investigated the function and localization of MreB in Caulobacter. Depletion of MreB results in the formation of lemon-shaped cells with cell wall defects. Caulobacter MreB forms two different types of spirals. Small spirals distributed over the entire cell length are present throughout the cell cycle. A more prominent spiral is formed at mid-cell in an FtsZ-dependent manner and disassembles after the completion of cell division. PBP2 immunofluorescence reveals spirals that resemble the small MreB spirals. As these structures are disturbed in an MreB depletion strain, MreB might serve as a tracking device for penicillin binding proteins.

Overexpression of CicA, another morphogene in Caulobacter, results in lemon-shaped cells that increase in volume by 10-fold (Fuchs et al., 2001). T. Fuchs (from U. Jenal's laboratory, Biozentrum Basel, Basel, Switzerland) has characterized suppressors that tolerated increased amounts of $\mathrm{CicA}$. These mutations mapped to three genes, acrA (CC0808), acrB (CC0807) and tolC (CC0806), encoding a multicomponent efflux system of unknown function. CicA was shown to associate with the outer membrane, which, together with its role in cell shape determination, may indicate that CicA is involved in cell wall biogenesis.

\section{Multicellular behaviour in Myxococcus xanthus}

Myxobacteria aggregate into multicellular complexes, termed fruiting bodies, upon starvation at high density on a solid surface. $M$. xanthus cells translocate by gliding over surfaces, either as individual cells using adventurous (A) motility or as a group using social (S) motility (Spormann, 1999). Mutations in one gene, $m g l A$, affect both motility systems. P. L. Hartzell (University of Idaho, Moscow, ID, USA) reported the characterization of a mutation that restores $S$ motility, but not $A$ motility, in an $m g l A$ mutant background. The mutation mapped to a putative tyrosine kinase, called MasK, which interacted with the MglA protein in a yeast two-hybrid assay. Increased amounts of fibrils (protein-associated cell surface polysaccharides) in the double mutant suggest that MasK is part of a fibril sensory system. In addition to fibrils, S motility relies on the production of type IV pili (McBride, 2001). Y. Li (W. Shi's laboratory at UCLA, Los Angeles, CA, USA) found a link between these two structural elements. Fibril synthesis mutants were hyperpiliated, and contact with fibrils on other cells or purified fibrils suppressed hyperpiliation, indicating that fibrils modulate pilus retraction. Pilus formation is blocked by mutations in the $\mathrm{tg} /$ gene that encodes a lipoprotein (Rodriguez-Soto and Kaiser, 1997). Pilus assembly and $\mathrm{S}$ motility can be restored transiently in $t g /$ mutant cells by mixing with $\operatorname{tg}^{+}$cells. E. D. Nudleman 
(D. Kaiser's laboratory at Stanford University, Stanford, CA, USA) showed that, in a mixed population, Tgl was physically transferred from $\mathrm{tgl}^{+}$to $\mathrm{tg}{ }^{-}$cells, which allowed the assembly of multimers of the outer membrane secretin PilQ and the polymerization of pili, thereby restoring $S$ motility. Similar protein transfer of the CglB lipoprotein between $c g / B^{+}$and $c g / B^{-}$cells resulted in the restoration of the pilus-independent $A$ motility. New genes implicated in A motility were identified by R. R. Yu (D. Kaiser's laboratory). The mutations mapped to loci encoding homologues of a glycosyltransferase, a mannosyltransferase, a GDP-mannose pyrophosphorylase and an exopolysaccharide biosynthesis gene, indicating that $A$ motility requires polysaccharide synthesis. Interestingly, all the mutants also showed a common defect in their ability to sense and respond to the orientation of agarose chains in the supporting medium (elasticotaxis; Fontes and Kaiser, 1999).

High-resolution motion analyses can provide valuable information on the complex behavioural patterns of developing M. xanthus cells. R. D. Welch (D. Kaiser's laboratory) recently characterized and modelled the coordinated cellular behaviour known as rippling (Welch and Kaiser, 2001). The relation between streaming and aggregation is now under investigation. Interestingly, streams do not always travel directly towards the nearest aggregation centre, but often to a more distant centre.

Chemotaxis is a prerequisite for the successful development of M. xanthus. D. R. Zusman (University of California, Berkeley, CA, USA) reported J. R. Kirby's research that characterized a new chemotaxis operon in which, intriguingly, the output signal is transcriptional regulation. In the che3 gene cluster, a $\sigma^{54}$-type response regulator, termed $\mathrm{CrdA}$, probably controls the regulation of several developmental genes (myxobacterial haemagglutinin expression was reduced in a $c r d A$ mutant). Generally, che3 operon mutants displayed developmental properties on nutrient medium. The advantage of chemosensory control of gene expression might be temporal regulation that depends on specific stimuli.

Another regulatory system in $M$. xanthus was identified recently (Guo et al., 2000). This three-component regulatory system integrates starvation and high-density signals to control spi (formerly designated 4521) expression. J. D. Rivera (H. B. Kaplan's laboratory, University of Texas Medical School, Houston, TX, USA) showed that these two stimuli are also likely to be transduced by an extracytoplasmic (ECF) sigma factor (ecfA) and its two cognate antisigma factors (reaA and reaB). This new regulatory system was discovered by transposon mutagenesis using a $\Omega 4445$-lac $Z$ transcriptional reporter screen. Interestingly, the absence of lipopolysaccharide (LPS) $\mathrm{O}$ antigen bypassed the high-density requirement for the expression of both spi and 4445 . H. B. Kaplan proposed that lack of LPS $\mathrm{O}$ antigen generates periplasmic stress that is sensed by the three-component system through SasS and through the sigma/antisigma pathway, thereby inducing spi and 4445 respectively. The $M$. xanthus starvation signal is, at least in part, an increase in (p)ppGpp accumulation. P. Brenner (M. H. Singer's laboratory, University of California, Davis, CA, USA) identified a novel regulator that controls expression of $\Omega 4469$, a gene regulated solely by ( $p$ )ppGpp levels. Mutations in gem1, which encodes a WD-repeat protein, resulted in accelerated development and increased $\Omega 4469$ expression.

At least five intercellular signals are required for fruiting body morphogenesis. S. Lobedanz (L. SøgaardAndersen's laboratory, University of Southern Denmark, Odense, Denmark) has resolved the prevailing controversy concerning the molecular nature of the $\mathrm{C}$-signal, the cell surface-associated signal that is transmitted by a contact-dependent mechanism. Purification of C-signal from developing $M$. xanthus cells showed that bona fide Csignal is identical to the $17 \mathrm{kDa}$ form of the CsgA protein. Recombinant CsgA protein corresponding to the C-terminal $17 \mathrm{kDa}$ of the entire $25 \mathrm{kDa}$ protein was purified and shown to have $\mathrm{C}$-signal activity. This protein does not bind $\mathrm{NAD}^{+}$in vitro and is likely to be generated by proteolytic cleavage. The synthesis of the $\mathrm{C}$-signal by proteolytic cleavage of the $25 \mathrm{kDa}$ CsgA protein should provide an additional checkpoint in the $M$. xanthus developmental programme.

\section{Differentiation and secondary metabolism in Streptomyces ssp.}

Streptomyces undergoes several developmental changes during its life cycle. Spore outgrowth leads to the formation of a substrate mycelium, which develops aerial hyphae that, in turn, allow sporulation. Specific genes can be attributed to each developmental stage. Mutations in the bld genes lead to a 'bald' phenotype, characterized by the absence of aerial hyphae. Mutations in the whi genes produce both cell types, but the aerial hyphae fail to complete sporulation, thereby retaining their initial 'white' colour (Chater, 2001).

M. A. Elliot (M. J. Buttner's laboratory at the John Innes Centre, Norwich, UK) performed a microarray analysis to determine genes that are differentially regulated in a bldN mutant compared with wild-type Streptomyces coelicolor. bldN encodes an ECF sigma factor that regulates a cascade of sigma factors and is also required for the transcription of the bldM gene encoding a member of the response regulator family. Several genes were identified that were expressed to a lesser extent in the bld $N$ and bldM mutant background. Some of these genes encode secreted proteins that localize 
to the surface of $S$. coelicolor, underscoring the important role played by the cell surface in aerial hyphae formation.

Aerial hyphae possess several surface layers that make them hydrophobic. One of these layers contains a mosaic of parallel rodlets. D. Claessen (L. Dijkhuizen's laboratory at the University of Groningen, Haren, The Netherlands) has identified two rodlet genes ( $r d l A$ and $r d l B)$ and shown that the corresponding proteins are localized to the outer surface of both aerial hyphae and spores (Claessen et al., 2002). Even though null mutations in these genes do not seem to affect aerial hyphae formation, they play an important role in attachment, which is instrumental for pathogenicity, e.g. in the potato pathogen S. scabies. Initiation of aerial hyphae formation depends on an extracellular signalling cascade of the following hierarchy: bld J $>K, L>A, H>G>\Delta \operatorname{ramR}>D, M>$ SapB. Secretion of extracellular factors allows unidirectional rescue of one bld mutant by another, a process called extracellular complementation (Willey et al., 1991). J. M. Willey (Hofstra University, Hempstead, NY, USA) demonstrated that a bldderived signalling molecule could be extracted from spent agar medium on which $S$. coelicolor bldC or bldG had been cultivated.

J. R. Nodwell (McMaster University, Hamilton, ON, Canada) elaborated the function of the ram (rapid aerial hyphae formation) gene cluster in spore-forming aerial hyphae formation. Expression of ramC, which encodes a membrane-associated serine/threonine kinase required for aerial hyphae formation, depends on other genes required for morphogenesis, including bldD, cprA and ram $R$. Whereas RamR, a response regulator, binds to the ramC promoter, BldD and CprA influence ramC expression indirectly. Interestingly, ramC is expressed in the substrate hyphae, but not in sporulating aerial hyphae (O'Connor et al., 2002). K. T. Nguyen (C. J. Thompson's laboratory, Biozentrum, University of Basel, Basel, Switzerland) demonstrated that overexpression of ramR restored the development and formation of the surfactant SapB in all classical bld strains. Disruption of the ramR gene prevented the synthesis of SapB and severely delayed development.

K. Chater (John Innes Centre, Norwich, UK) reported on the action of $b / d A$ and $b / d H$. The $b / d A$ gene product is the only tRNA cognate with UUA, the rarest codon in Streptomyces. BldH is the $S$. coelicolor equivalent of a gene that was previously characterized in $S$. griseus as the key regulatory gene through which the quorum-indicating $\gamma$-butyrolactone A-factor exerts its effects on both secondary metabolism and normal morphological development (Ohnishi et al., 1999). bldA seems to exert its effect on morphological development but, interestingly, not on secondary metabolism through a UUA codon in the bldH mRNA. Thus, Streptomyces spp. differ in the signals and targets that regulate developmental processes. K. Chater and coworkers have also identified a key gene for the synthesis of the volatile earth smell of Streptomyces (geosmin), and preliminary data indicate that geosmin or a related compound may function as a signalling molecule for the development of $S$. coelicolor.

Streptomyces hyphae contain several chromosomes that are separated upon cell division during spore formation. K. Flärdh (Uppsala University, Uppsala, Sweden) described the isolation and characterization of an FtsZ mutant (A249T) that divides normally when growing as vegetative hyphae (albeit with a reduced frequency), but failed to assemble correct $Z$ rings and to divide during sporulation, indicating that Z-ring formation may be controlled developmentally (Grantcharova et al., 2002). K. Flärdh also showed that a homologue of $B$. subtilis cell division protein DivIVA plays an important role in tip growth and morphogenesis of the hyphae of $S$. coelicolor. A DivIVA-EGFP fusion localized distinctively at vegetative hyphal tips and nascent lateral branch points. A decrease in the expression of the essential divIVA gene led to irregularly shaped, crooked hyphae, which often branched in the apical cell. This phenotype is reminiscent of many tip growth and nuclear migration mutants in filamentous fungi. Increasing DivIVA amounts in the cell gave rise to rounded and swollen pear-shaped cells rather than hyphae. Thus, Streptomyces DivIVA has a strong impact on cell shape determination and does not function primarily in cell division. It is the first Streptomyces protein found to be targeted to the growing hyphal tips.

So far, S. coelicolor has primarily been studied on solid substrates. G. van Keulen (H. A. Wösten's laboratory, University of Groningen, Haren, The Netherlands) has initiated a study in standing liquid cultures. Surprisingly, S. coelicolor, classified as an aerobic organism, grew at the air-liquid interface even though the cultures became completely anoxic within 1 week. Colonies were surrounded by a rigid protein film. The $S$. coelicolor genome contains genes encoding gas vesicle proteins, which is unexpected for a soil organism. Nevertheless, electron microscopy revealed the presence of gas vesicles in liquid cultures.

\section{Acknowledgements}

We thank the Department of Energy (DE-FG02-02ER63409) and the American Society for Microbiology for supporting the meeting. The authors are grateful to Heidi Kaplan (University of Texas, Houston), Jeff Errington (Oxford), Justin Nodwell (McMaster University, Hamilton), Klas Flärdh (Uppsala University) and Beth Lazazzera (UCLA) for input on the manuscript. R.M.F. acknowledges the receipt of an EMBO longterm fellowship. Work in our laboratory (J.W.G.) is supported by grants from the National Institutes of Health (GM48417) and the National Science Foundation (MCB-9986127). 


\section{References}

Bentley, S.D., Chater, K.F., Cerdeno-Tarraga, A.M., Challis, G.L., Thomson, N.R., James, K.D., et al. (2002) Complete genome sequence of the model actinomycete Steptomyces coelicolor A3(2). Nature 417: 141-147.

Ben-Yehuda, S., and Losick, R. (2002) Asymmetric cell division in $B$. subtilis involves a spiral-like intermediate of the cytokinetic protein FtsZ. Cell 109: 257-266.

Branda, S.S., Gonzalez-Pastor, J.E., Ben-Yehuda, S., Losick, R., and Kolter, R. (2001) Fruiting body formation by BacilIus subtilis. Proc Natl Acad Sci USA 98: 11621-11626.

Burkholder, W.F., and Grossman, A.D. (2000) Regulation of the initiation of endospore formation in Bacillus subtilis. In Prokaryotic Development. Brun, Y.V., and Shimkets, L.J. (eds). Washington, DC: American Society for Microbiology Press, pp. 151-166.

Chater, K.F. (2001) Regulation of sporulation in Streptomyces coelicolor A3(2): a checkpoint multiplex? Curr Opin Microbiol 4: 667-673.

Claessen, D., Wosten, H.A., Keulen, Gv.G., Faber, O.G., Alves, A.M., Meijer, W.G., and Dijkhuizen, L. (2002) Two novel homologous proteins of Streptomyces coelicolor and Streptomyces lividans are involved in the formation of the rodlet layer and mediate attachment to a hydrophobic surface. Mol Microbiol 44: 1483-1492.

Draper, G.C., and Gober, J.W. (2001) Bacterial chromosome segregation. Annu Rev Microbiol 56: 567-597.

Easter, J., Jr, and Gober, J.W. (2002) ParB-stimulated nucleotide exchange regulates a switch in functionally distinct ParA activities. Mol Cell 2: 427-434.

England, J.C., and Gober, J.W. (2001) Cell cycle control of cell morphogenesis in Caulobacter. Curr Opin Microbiol 4: 674-680.

van den Ent, F., Amos, L.A., and Lowe, J. (2001) Prokaryotic origin of the actin cytoskeleton. Nature 413: 39-44.

Fontes, M., and Kaiser, D. (1999) Myxococcus cells respond to elastic forces in their substrate. Proc Natl Acad Sci USA 96: 8052-8057.

Fuchs, T., Wiget, P., Osteras, M., and Jenal, U. (2001) Precise amounts of a novel member of a phosphotransferase superfamily are essential for growth and normal morphology in Caulobacter crescentus. Mol Microbiol 39: 679-692.

Grantcharova, N., Ubhayasekera, W., Mowbray, S.L., McCormick, J.R., and Flärdh, K. (2002) A missense mutation in fts $Z$ differentially affects vegetative and developmentally controlled cell division in Streptomyces coelicolor A3(2). Mol Microbiol 47: 645-656.

Guo, D., Wu, Y., and Kaplan, H.B. (2000) Identification and characterization of genes required for early Myxococcus xanthus developmental gene expression. J Bacteriol 182: 4564-4571.

Hinz, A.J., Larson, D.E., Smith, C.S., and Brun, Y.V. (2002) The Caulobacter crescentus polar organelle development protein PodJ is differentially localized and is required for polar targeting of the PleC development regulator. Mol Microbiol 47: 929-941.

Hu, Z., Gogol, E.P., and Lutkenhaus, J. (2002) Dynamic assembly of MinD on phospholipid vesicles regulated by ATP and MinE. Proc Natl Acad Sci USA 99: 6761-6766.
Ishikawa, S., Core, L., and Perego, M. (2002) Biochemical characterization of aspartyl phosphate phosphatase interaction with a phosphorylated response regulator and its inhibition by a pentapeptide. J Biol Chem 277: 2048320489.

Jensen, R.B., Wang, S.C., and Shapiro, L. (2002) Dynamic localization of proteins and DNA during a bacterial cell cycle. Nature Rev Mol Cell Biol 3: 167-176.

Jones, L.J., Carballido-Lopez, R., and Errington, J. (2001) Control of cell shape in bacteria: helical, actin-like filaments in Bacillus subtilis. Cell 104: 913-922.

Kunkel, B., Losick, R., and Stragier, P. (1990) The Bacillus subtilis gene for the development transcription factor sigma $\mathrm{K}$ is generated by excision of a dispensable DNA element containing a sporulation recombinase gene. Genes Dev 4: 525-535.

Kunst, F., Ogasawara, N., Moszer, I., Albertini, A.M., Alloni, G., Azevedo, V., et al. (1997) The complete genome sequence of the gram-positive bacterium Bacillus subtilis. Nature 390: 249-256.

Laub, M.T., Chen, S.L., Shapiro, L., and McAdams, H.H. (2002) Genes directly controlled by CtrA, a master regulator of the Caulobacter cell cycle. Proc Natl Acad Sci USA 99: 4632-4637.

Levin, P.A., and Losick, R. (1996) Transcription factor Spo0A switches the localization of the cell division protein FtsZ from a medial to a bipolar pattern in Bacillus subtilis. Genes Dev 10: 478-488.

McBride, M.J. (2001) Bacterial gliding motility: multiple mechanisms for cell movement over surfaces. Annu Rev Microbiol 55: 49-75.

Margolin, W. (2001) Spatial regulation of cytokinesis in bacteria. Curr Opin Microbiol 4: 647-652.

Mohl, D.A., and Gober, J.W. (1997) Cell cycle-dependent polar localization of chromosome partitioning proteins in Caulobacter crescentus. Cell 88: 675-684.

Mohr, C.D., MacKichan, J.K., and Shapiro, L. (1998) A membrane-associated protein, FliX, is required for an early step in Caulobacter flagellar assembly. $J$ Bacteriol 180: 21752185.

Møller-Jensen, J., Jensen, R.B., Lowe, J., and Gerdes, K. (2002) Prokaryotic DNA segregation by an actin-like filament. EMBO J 21: 3119-3127.

Muir, R.E., and Gober, J.W. (2001) Regulation of late flagellar gene transcription and cell division by flagellum assembly in Caulobacter crescentus. Mol Microbiol 41: 117-130.

Muir, R.E., O'Brien, T.M., and Gober, J.W. (2001) The Caulobacter crescentus flagellar gene, fliX, encodes a novel trans-acting factor that couples flagellar assembly to transcription. Mol Microbiol 39: 1623-1637.

Nierman, W.C., Feldblyum, T.V., Paulsen, I.T., Nelson, K.E., Eisen, J., Heidelberg, J.F., et al. (2001) Complete genome sequence of Caulobacter crescentus. Proc Natl Acad Sci USA 98: 4136-4141.

O'Connor, T.J., Kanellis, P., and Nodwell, J.R. (2002) The ram $C$ gene is required for morphogenesis in Streptomyces coelicolor and expressed in a cell type-specific manner under the direct control of RamR. Mol Microbiol 45: 45-57.

Ohnishi, Y., Kameyama, S., Onaka, H., and Horinouchi, S. (1999) The A-factor regulatory cascade leading to strepto- 
mycin biosynthesis in Streptomyces griseus: identification of a target gene of the A-factor receptor. Mol Microbiol 34: 102-111.

Perego, M., and Brannigan, J.A. (2001) Pentapeptide regulation of aspartyl-phosphate phosphatases. Peptides 22: 1541-1547.

Rodriguez-Soto, J.P., and Kaiser, D. (1997) The tgl gene: social motility and stimulation in Myxococcus xanthus. $J$ Bacteriol 179: 4361-4371.

Spormann, A.M. (1999) Gliding motility in bacteria: insights from studies of Myxococcus xanthus. Microbiol Mol Biol Rev 63: 621-641.

Viollier, P.H., Sternheim, N., and Shapiro, L. (2002) Identification of a localization factor for the polar positioning of bacterial structural and regulatory proteins. Proc Natl Acad Sci USA 99: 13831-13836.

Welch, R., and Kaiser, D. (2001) Cell behavior in traveling wave patterns of myxobacteria. Proc Natl Acad Sci USA 98: 14907-14912.

Whiteley, M., Bangera, M.G., Bumgarner, R.E., Parsek, M.R., Teitzel, G.M., Lory, S., and Greenberg, E.P. (2001) Gene expression in Pseudomonas aeruginosa biofilms. Nature 413: $860-864$.

Willey, J., Santamaria, R., Guijarro, J., Geistlich, M., and Losick, R. (1991) Extracellular complementation of a developmental mutation implicates a small sporulation protein in aerial mycelium formation by $S$. coelicolor. Cell 65: 641650. 